\title{
Tratamentos medicamentosos para acne vulgar em adolescentes e jovens adultos
}

\author{
Drug treatments for acne vulgaris in adolescents and young adults \\ Tratamientos farmacológicos para el acné vulgar en adolescentes y adultos jóvenes
}

Recebido: 15/04/2021 | Revisado: 20/04/2021 | Aceito: 26/04/2021 | Publicado: 10/05/2021

Giovanna Stefanne Lópes Barbosa

ORCID: https://orcid.org/0000-0003-3387-1219

Universidade Federal do Delta do Parnaíba, Brasil

E-mail: gistefanne@gmail.com

Camila Pereira Miranda Costa

ORCID: https://orcid.org/0000-0002-4724-6943

Universidade Federal do Delta do Parnaíba, Brasil

E-mail: camilapcosta319@gmail.com

Mariana Veras Rocha Borges

ORCID: https://orcid.org/0000-0003-2922-4377

Universidade Federal do Delta do Parnaíba, Brasil

E-mail: mariana_vrborges@hotmail.com

Marinice Saraiva Attem

ORCID: https://orcid.org/0000-0002-7460-5788 Instituto de Educação Superior Vale do Parnaíba, Brasil E-mail: mariniceattem@gmail.com

Bianca Lopes Cacau

ORCID: https://orcid.org/0000-0002-0525-8073

Universidade Federal do Delta do Parnaíba, Brasil

E-mail: bianca.lopescacau1@gmail.com

Maria Simone Lopes

ORCID: https://orcid.org/0000-0003-2689-3655

Universidade Federal do Delta do Parnaíba, Brasil

E-mail: simonemaia617@gmail.com

Daniela Winckler Mass

ORCID: https://orcid.org/0000-0003-3655-4040

Universidade Federal do Delta do Parnaíba, Brasil

E-mail: danielamasspi@gmail.com

Breno Serafim Pereira

ORCID: https://orcid.org/0000-0001-5441-9198

Universidade Federal do Delta do Parnaíba, Brasil

E-mail: breno-serafim@hotmail.com

Mary Ângela de Oliveira Canuto Coutinho

ORCID: https://orcid.org/0000-0003-2157-4794

Universidade Federal do Delta do Parnaíba, Brasil

E-mail: maryangelacanuto@gmail.com

Fernando Aguiar Luz

ORCID: https://orcid.org/0000-0002-6625-5941

Universidade Federal do Delta do Parnaíba, Brasil

E-mail: biopele.fernandoluz@hotmail.com

Karina Rodrigues dos Santos

ORCID: https://orcid.org/0000-0003-2872-0002

Universidade Federal do Delta do Parnaíba, Brasil

E-mail: krsantos2004@yahoo.com.br

Ludmilla Figueiredo Vale Fontenelle

ORCID: https://orcid.org/0000-0003-4885-8340

Universidade Federal do Delta do Parnaíba, Brasil E-mail: lufigueiredo@hotmail.com

\begin{abstract}
Resumo
Objetivo: Descrever os tratamentos medicamentosos disponíveis para acne vulgar em adolescentes e jovens adultos. Metodologia: trata-se de uma revisão integrativa, com buscas nas bases de dados: Medical Literature Analysis and Retrieval System (MEDLINE/PubMed), Scientific Electronic Library Online (SciELO) e Literatura Latino-Americana e do Caribe em Ciências da Saúde (LILACS), utilizando os Descritores em Ciências da Saúde (DECS): "acne vulgar", "terapêutica", “adolescentes" e "adulto jovem", combinados por meio de operadores boleanos, do período de 2015 a 2020. Resultados: foram selecionados 15 artigos. Por se tratar de doença de etiologia multifatorial, diversas terapêuticas precisam ser adotadas para o tratamento eficaz. Os tratamentos disponíveis envolvem retinóides, antibióticos, corticoides, peróxido de benzó́la, adapaleno, anticoncepcionais orais, formulações de extratos naturais, cepas de
\end{abstract}


bactérias, nanopartículas, incluindo tratamentos tópicos e sistêmicos. Conclusão: os tratamentos para acne vulgar disponíveis são múltiplos e variados, e têm sido aprimorados e ainda mais diversificados, entretanto, ressalva-se a necessidade de mais estudos científicos para desenvolver tratamentos eficazes e com menos efeitos colaterais, contribuindo para a acessibilidade e maior adesão dos acometidos.

Palavras-chave: Acne vulgar; Terapêutica; Adolescentes.

\begin{abstract}
Objective: To describe the drug treatments available for acne vulgaris in adolescents and young adults. Methodology: this is an integrative review, with searches in the databases: Medical Literature Analysis and Retrieval System (MEDLINE/PubMed), Scientific Electronic Library Online (SciELO) and Latin American and Caribbean Literature in Health Sciences (LILACS), using Health Sciences Descriptors (DECS): "acne vulgar", "terapêutica", "adolescentes" e "adulto jovem", combined through Boolean operators, from 2015 to 2020 . Results: Were selected 15 articles. Because it is a disease of multifactorial etiology, several therapies need to be adopted for effective treatment. The available treatments involve retinoids, antibiotics, corticosteroids, benzoyl peroxide, adapalene, oral contraceptives, formulations of natural extracts, strains of bacteria, nanoparticles, including topical and systemic treatments. Conclusion: the treatments for acne vulgaris are multiple and varied, and have been improved and even more diversified, however, the need for more scientific studies to develop effective treatments with fewer side effects is emphasized, contributing to accessibility and greater adherence affected.
\end{abstract}

Keywords: Acne vulgaris; Therapeutics; Adolescent.

\title{
Resumen
}

Objetivo: Describir los tratamientos farmacológicos disponibles para el acné vulgar en adolescentes y adultos jóvenes. Metodología: se trata de una revisión integradora, con búsquedas en las bases de datos: Sistema de Análisis y Recuperación de Literatura Médica (MEDLINE/PubMed), Biblioteca Electrónica Científica en Línea (SciELO) y Literatura Latinoamericana y del Caribe en Ciencias de la Salud (LILACS), utilizando Descriptores de Ciencias de la Salud (DECS): "acne vulgar", "terapêutica", "adolescentes" e "adulto jovem", combinados a través de operadores booleanos, de 2015 a 2020. Resultados: Se seleccionaron 15 artículos. Como se trata de una enfermedad de etiología multifactorial, es necesario adoptar varias terapias para lograr un tratamiento eficaz. Los tratamientos disponibles incluyen retinoides, antibióticos, corticosteroides, peróxido de benzoilo, adapaleno, anticonceptivos orales, formulaciones de extractos naturales, cepas de bacterias, nanopartículas, incluidos tratamientos tópicos y sistémicos. Conclusión: los tratamientos para el acné vulgar son múltiples y variados, y se han mejorado e incluso más diversificado, sin embargo, se enfatiza la necesidad de más estudios científicos para desarrollar tratamientos efectivos con menos efectos secundarios, contribuyendo a la accesibilidad y mayor adherencia afectada.

Palabras clave: Acné vulgar; Terapéutica; Adolescente.

\section{Introdução}

A adolescência, fase que geralmente compreende a puberdade, é um período em que ocorrem intensas mudanças biológicas e psicológicas no indivíduo e situa-se na faixa etária de 13 a 18 anos. Já a fase de adulto jovem, período de transição da adolescência para a adultez, inicia-se a partir de 18 anos. O adulto jovem pode ser compreendido na faixa de 19 a 24 anos (NCBI, 2009; Bagatin et al., 2014). Uma afecção bastante comum em ambos os períodos é a acne, alcançando uma incidência de cerca de $85 \%$ em adolescentes, e persistindo em $50 \%$ na idade adulta, por inúmeros fatores, dentre eles por ser uma fase de importantes alterações hormonais. O tratamento para acne se faz importante, pois as lesões, se negligenciadas, podem deixar cicatrizes permanentes que repercutem na esfera psicossocial do indivíduo (Montagner \& Costa, 2010; Silva \& Pereira, 2018; Lima, Barros, \& Lúcio, 2020).

A acne vulgar é uma doença dermatológica crônica que ocorre devido a um processo inflamatório - exceto na acne comedoniana, que consiste na obstrução por queratina e sebo - no folículo pilossebáceo. A pele é composta por duas camadas. A camada mais superficial é chamada de epiderme, e abaixo da epiderme está a derme (Almeida-Junior et al., 2020). Na derme encontra-se a glândula sebácea, produtora de uma secreção de natureza lipídica, que geralmente desemboca em um folículo piloso. Essa unidade, denominada pilossebácea, localiza-se por todo o corpo em diferentes proporções, com exceção das palmas das mãos e plantas dos pés, e predomina no couro cabeludo, fronte, região interescapular e anogenital (Costa \& Velho, 2018).

Além de ser crônica, a acne vulgar é de origem multifatorial. Entre os principais fatores estão: produção exacerbada de secreção pelas glândulas sebáceas estimuladas por hormônios, hiperqueratose folicular, proliferação bacteriana e inflamação. 
Outras causas também podem ser citadas, tais como genética, uso de medicamentos, cosméticos ricos em óleo mineral e estresse (Silva \& Pereira, 2018). Clinicamente, pode ser classificada em não-inflamatória ou inflamatória (Saraiva et al., 2020). Uma outra forma de classificação leva em consideração o grau de intensidade das lesões, variando do I ao V, compreendendo comedões, pápulas, pústulas e lesões nódulo-císticas, que podem evoluir ainda para abscessos e cistos (Rodrigues, Leite, Yoshida, Carneiro, \& Santos, 2019).

$\mathrm{Na}$ acne grau I, comedoniana, não há inflamação, e as lesões são caracterizadas por tampões de queratina que se formam dentro dos óstios dos folículos, podendo ser abertos - comedões pretos - ou fechados - comedões brancos. À medida que o processo inflamatório surge e avança, irrompem outras lesões que caracterizam os demais graus da acne. Na acne grau II, além dos comedões, há lesões pápulo-pustulosas. Na acne grau III nota-se a presença de pápulas, pústulas, nódulos e cistos. Na acne grau IV, também chamada de conglobata, a inflamação se dá de forma mais exuberante com nódulos inflamatórios, abcessos e fístulas. $\mathrm{O}$ grau $\mathrm{V}$, acne fulminante, a forma mais severa e rara, caracteriza-se pelo acometimento sistêmico com surgimento agudo de nódulos inflamatórios e crostas hemorrágicas, febre, leucocitose e necrose (Rodrigues et al., 2019).

A epidemiologia de acne é bastante abrangente, acometendo diferentes raças, sexo - com discreto predomínio no sexo feminino, porém geralmente mais grave no sexo masculino - e idades, iniciando na adolescência. Contudo, apesar de seu início inflamatório ocorrer comumente na adolescência, no período da puberdade, devido aos diferentes fatores listados anteriormente, a acne pode se estender à fase adulta e acarretar transtornos psicossociais e sequelas locais aos pacientes (Costa, Sousa, Conradesque, Carniel, \& Zago, 2020).

A busca por tratamento é importante, não apenas por fatores estéticos, mas também pelas repercussões psicológicas. O paciente com acne poderá desenvolver problemas como baixa autoestima, insegurança, timidez e infelicidade, sentimentos que podem lhe acompanhar por toda a vida. Assim sendo, a acne vulgar deve ser tratada o mais precocemente possível com o intuito de evitar tanto as cicatrizes físicas quanto as emocionais (Santos, Martins \& Perez, 2019).

O tratamento dependerá das manifestações clínicas apresentadas pelo paciente. Dessa forma, o dermatologista pode optar por tratamentos tópicos como cremes ou loções, ou tratamentos orais à base de antibióticos, anticoncepcionais, retinóides, entre outros. A isotretinoína é um retinóide oral normalmente prescrito para pacientes que não obtiveram boa resposta terapêutica a outros métodos, bem como para casos mais graves (Soyuduru, Adişen, Özer, \& Aksakal, 2019; Lima et al., 2020). Ressalta-se ainda que a adesão ao tratamento revela-se como um problema, principalmente em relação aos tratamentos tópicos, devido aos efeitos colaterais e ao tempo prolongado de utilização (Dikicier, 2019).

Durante o período de tratamento farmacológico, algumas práticas são recomendadas, auxiliando no combate à afecção, como: higienização da área acometida pela acne (normalmente o rosto) ao menos duas vezes ao dia com sabão adequado; não realizar a extração de comedões e demais lesões sem o auxílio do profissional habilitado e evitar exposição ao sol com a pele desprotegida (Silva et al., 2020).

Diante do exposto, tem-se como objetivo do presente trabalho descrever os tratamentos medicamentosos disponíveis para acne vulgar em adolescentes e jovens adultos.

\section{Metodologia}

Foi realizada uma revisão integrativa, estudo que agrupa, analisa e sumariza os achados de pesquisas sobre determinado tema. Tendo em vista a exponencial produção de conhecimento científico na área da saúde, houve a necessidade do desenvolvimento de mecanismos aplicados à pesquisa científica, com etapas metodológicas, de forma a proporcionar aos profissionais da área um melhor uso das evidências elucidadas nos diversos estudos (Garcia, Fonseca, Aroni, \& Galvão, 2016). Nesse sentido, a revisão integrativa tem sido caracterizada como uma ferramenta ímpar no campo da saúde, visto que consegue elencar de forma concisa os inúmeros conhecimentos disponíveis acerca de determinada temática, direcionando a prática 
fundamentada em conhecimento científico embasado (Souza, Silva, \& Carvalho, 2010).

Esta revisão integrativa tem como pergunta norteadora "Quais os tratamentos disponíveis para acne vulgar em adolescentes e jovens adultos?”. As buscas para elucidar esta questão foram realizadas nas seguintes bases de dados: Medical Literature Analysis and Retrieval System (MEDLINE/PubMed), Scientific Electronic Library Online (SciELO) e Literatura Latino-Americana e do Caribe em Ciências da Saúde (LILACS), sendo considerado o período de 2015 a 2020. Na base PubMed foram utilizados os Medical Subject Headings (MeSH): "acne vulgaris", "therapeutics", "adolescents" e "young adult"; e nas bases SciELO e LILACS foram usados os Descritores em Ciências da Saúde (DECS): "acne vulgar", "terapêutica", "adolescentes" e "adulto jovem" e seus correspondentes em língua inglesa, combinados por meio de operadores boleanos, seguindo as estratégias de busca:

a. "acne vulgar" AND terapêutica AND (adolescentes OR "adulto jovem")

b. $\quad$ "acne vulgaris" AND therapeutics AND (adolescents OR "young adult")

Foram considerados critérios de inclusão: artigos em inglês ou português, publicados dentro do período estipulado, com texto completo disponível, fontes primárias de dados e relacionados à pergunta da pesquisa. Os critérios de exclusão foram: artigos publicados em outros idiomas que não inglês ou português, artigos publicados fora do intervalo de tempo determinado, revisões, artigos baseados em opiniões de especialistas, não disponíveis na íntegra e que não se relacionavam com a pergunta desta pesquisa. Na Figura 1 é possível observar o método para a seleção dos artigos. 
Figura 1 - Seleção dos artigos para a revisão de literatura.

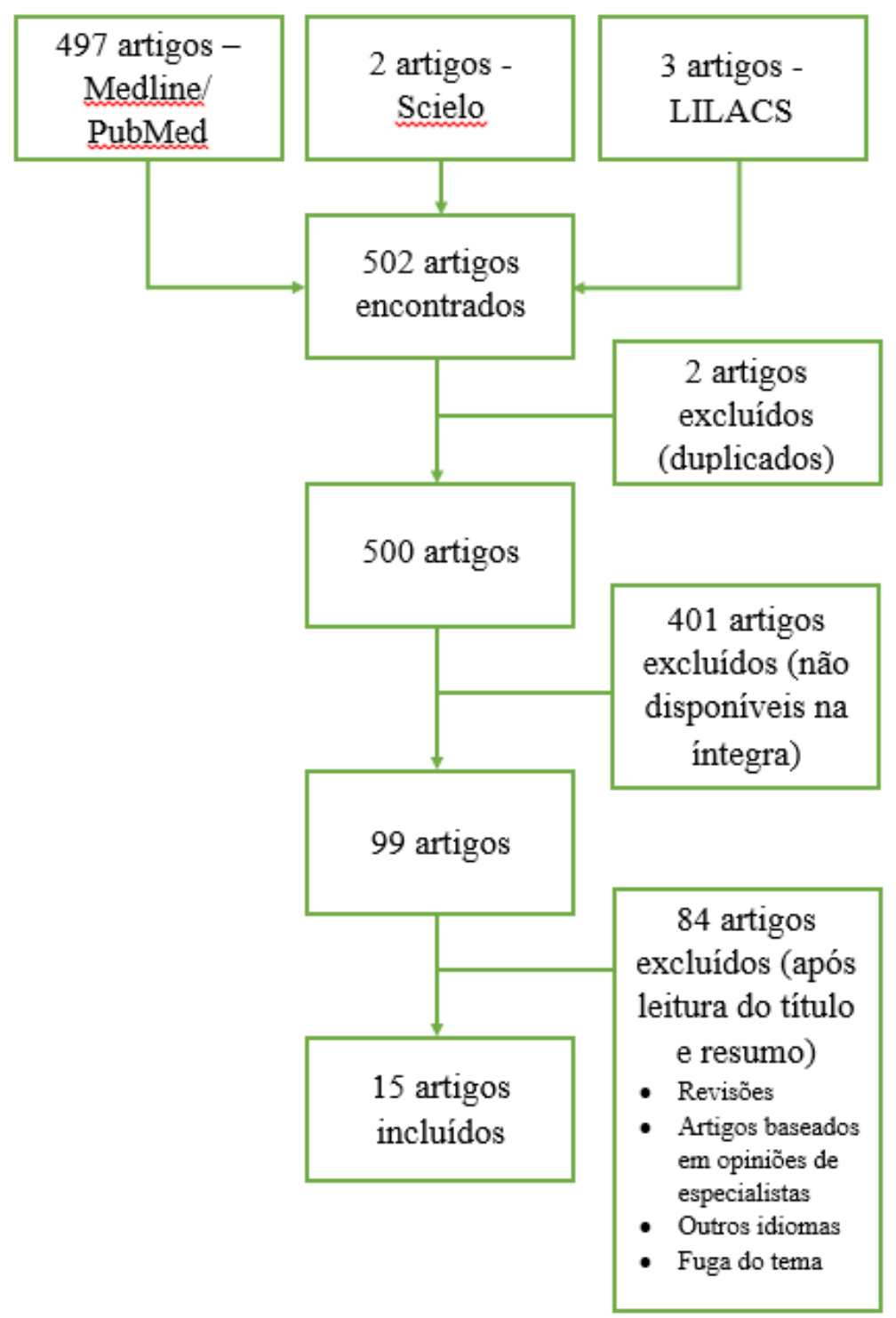

Fonte: Barbosa et al. (2021).

Diante da metodologia apresentada, foram incluídos 15 artigos na presente revisão, os quais foram tabulados quanto autoria, ano de publicação, objetivo, tratamentos empregados e principais resultados, utilizando o software Microsoft Excel® 2016.

\section{Resultados}

Os artigos incluídos na revisão foram sintetizados no Quadro 1, sendo mencionados os autores, ano de publicação, $\mathrm{n}^{\circ}$ de participantes, objetivos, tratamentos empregados e os principais achados. 
Research, Society and Development, v. 10, n. 4, e39010515094, 2021

(CC BY 4.0) | ISSN 2525-3409 | DOI: http://dx.doi.org/10.33448/rsd-v10i5.15094

Quadro 1 - Artigos incluídos na revisão. Parnaíba, 2020.

\begin{tabular}{|c|c|c|c|c|}
\hline $\begin{array}{l}\text { Autores (ano de } \\
\text { publicação) }\end{array}$ & $\begin{array}{l}\mathbf{N}^{\circ} \text { de } \\
\text { partici- } \\
\text { pantes }\end{array}$ & Objetivos & $\begin{array}{l}\text { Tratamentos } \\
\text { empregados }\end{array}$ & Principais achados \\
\hline Dikicier (2019) & 250 & $\begin{array}{l}\text { Avaliar a eficácia do tratamento tópico para acne } \\
\text { e a adesão dos pacientes. }\end{array}$ & $\begin{array}{l}\text { Tópicos: antibióticos, } \\
\text { retinóides, peróxido de } \\
\text { benzoíla, ácido salicílico }\end{array}$ & $\begin{array}{l}\text { De todos os pacientes incluídos, } 45,6 \% \text { desistiram da terapia por dois motivos: } \\
\text { falta de resposta }(62,3 \%) \text { e efeitos colaterais }(37,7 \%) \text {. Tratamentos } \\
\text { antibacterianos resultaram em maior falta de resposta, mas a taxa de efeitos } \\
\text { colaterais foi menor. A descontinuação devido à falta de resposta foi maior em } \\
\text { pacientes com acne grave. Os efeitos colaterais foram maiores em pacientes com } \\
\text { acne comedoniana. }\end{array}$ \\
\hline $\begin{array}{l}\text { Eichenfield, Sugarman, } \\
\text { Guenin, Harris e Bhatt } \\
\text { (2019) }\end{array}$ & 154 & $\begin{array}{c}\text { Avaliar a segurança e eficácia de uma nova loção } \\
\text { de tretinoína } 0,05 \%, \text { uma vez ao dia em } \\
\text { indivíduos pré-adolescentes ( } \leq 13 \text { anos) com acne } \\
\text { moderada a grave. }\end{array}$ & $\begin{array}{l}\text { Loção de tretinoina } \\
\qquad, 05 \% \text {. }\end{array}$ & $\begin{array}{l}\text { Na semana } 12 \text {, a redução percentual média na contagem de lesões inflamatórias } \\
\text { e não inflamatórias foi de } 49,5 \% \text { e } 44,0 \% \text {, respectivamente, em comparação a } \\
\text { uma redução percentual de } 31,4 \% \text { e } 18,8 \% \text { no grupo controle (ambos p = 0,001). } \\
\text { O sucesso do tratamento foi alcançado por } 23,7 \% \text { dos indivíduos na } 12^{\mathrm{a}} \text { semana, } \\
\text { em comparação com } 7,2 \% \text { do grupo controle }(\mathrm{p}=0,009) \text {. }\end{array}$ \\
\hline Fuchs et al. (2019) & 570 & $\begin{array}{l}\text { Estabelecer uma correlação entre a ingestão de } \\
\text { diferentes tipos de medicamentos } \\
\text { antiandrogênicos e a intensidade do hirsutismo, } \\
\text { acne, intensidade de dor menstrual e sexualidade. }\end{array}$ & $\begin{array}{l}\text { Contraceptivos orais } \\
\text { hormonais }\end{array}$ & $\begin{array}{l}\text { A redução da intensidade da acne foi estatisticamente significativa, após três } \\
\text { meses de tratamento com anticoncepcionais em todos os grupos estudados. }\end{array}$ \\
\hline Karoglan et al. (2019) & 14 & $\begin{array}{c}\text { Reunir mais evidências sobre a viabilidade, } \\
\text { segurança e eficácia da aplicação tópica de cepas } \\
\text { benéficas de Propionibacterium acnes em } \\
\text { pacientes com pele propensa a acne. }\end{array}$ & $\begin{array}{c}\text { Modulação biológica com } \\
\text { cepas de } P \text {. acnes não } \\
\text { causadoras de acne }\end{array}$ & $\begin{array}{l}\text { A composição da microbiota da pele mudou para as formulaçães utilizadas no } \\
\text { estudo. Não foram observados eventos adversos indesejáveis, irritação visível ou } \\
\text { flare up significativo. Houve diminuição do pH da pele. O número de lesões } \\
\text { não-inflamatórias diminuiu, entretanto não houve alteração daquelas } \\
\text { inflamatórias. A contagem de comedões melhorou clinicamente. }\end{array}$ \\
\hline Soyuduru et al. (2019) & 58 & $\begin{array}{l}\text { Avaliar a associação de resistência à insulina e } \\
\text { níveis de adipocitocina em pacientes com acne } \\
\text { vulgar usando como tratamento a isotretinoína. } \\
\text { Busca-se avaliar os efeitos da isotretinoína nos } \\
\text { adipócitos e suas implicações inflamatórias. }\end{array}$ & Isotretinoína & $\begin{array}{l}\text { O estudo sugere que o tratamento de acne vulgar com isotretinoína causa } \\
\text { resistência à insulina nesses pacientes, independentemente da idade, IMC, } \\
\text { quantidade de gordura corporal e níveis lipídicos desses pacientes. }\end{array}$ \\
\hline $\begin{array}{l}\text { Pavithra, Upadya e Rukmini } \\
\text { (2019) }\end{array}$ & 84 & $\begin{array}{c}\text { Comparar a eficácia de uma dieta de baixa carga } \\
\text { glicêmica associada a gel tópico de peróxido de } \\
\text { benzoíla 2,5\% com apenas o gel tópico de } \\
\text { peróxido de benzoíla 2,5\% nos graus } 1,2 \text { e } 3 \text { de } \\
\text { acne vulgar. }\end{array}$ & $\begin{array}{l}\text { Peróxido de benzoila } \\
\text { tópico } 2,5 \% \text { gel } \\
\text { (associado a dieta } \\
\text { hipoglicêmica) }\end{array}$ & $\begin{array}{c}\text { Ambos os grupos mostraram redução na contagem de acne na } 12^{\mathrm{a}} \text { semana, mas } \\
\text { sem diferença estatística entre eles. }\end{array}$ \\
\hline
\end{tabular}


Research, Society and Development, v. 10, n. 4, e39010515094, 2021

(CC BY 4.0) | ISSN 2525-3409 | DOI: http://dx.doi.org/10.33448/rsd-v10i5.15094

\begin{tabular}{|c|c|c|c|c|}
\hline $\begin{array}{l}\text { Monfrecola, Capasso, Russo } \\
\text { e Fabbrocini (2018) }\end{array}$ & 40 & $\begin{array}{c}\text { Avaliar os efeitos clínicos reais de um novo } \\
\text { creme facial seletivo para UV (Acne RA -1,2, } \\
\text { Meda Pharma, Solna, Suécia) na acne, sua função } \\
\text { de barreira epidérmica, produção de sebo, } \\
\text { aderência e tolerabilidade quando usado em } \\
\text { conjunto com tratamento farmacológico da acne. }\end{array}$ & $\begin{array}{l}\text { Novo creme facial } \\
\text { seletivo para UV (Acne } \\
\text { RA-1,2, Meda Pharma, } \\
\text { Solna, Suécia }\end{array}$ & $\begin{array}{l}\text { Acne RA-1,2 em combinação com o tratamento farmacológico da acne mostrou } \\
\text { melhora clínica da acne, da função de barreira epidérmica e redução na produção } \\
\text { de sebo. }\end{array}$ \\
\hline $\begin{array}{l}\text { Gupta, Timothy, Sopkovich, } \\
\text { Massick e Kaffenberger } \\
\text { (2017) }\end{array}$ & 216 & $\begin{array}{l}\text { Comparar a falha do tratamento, definida como } \\
\text { progressão para isotretinoína 5, entre as } \\
\text { formulações de minociclina ERM (liberação } \\
\text { estendida) e GM (genérico 2x ao dia) e comparar } \\
\text { a carga administrativa (ligações para pacientes, } \\
\text { mensagens para farmácias) entre essas duas } \\
\text { formulações. }\end{array}$ & Minociclina & $\begin{array}{l}\text { Embora ERM possa ser mais conveniente para os pacientes, não pareceu } \\
\text { oferecer uma vantagem clínica significativa sobre o GM. A duração média entre } \\
\text { a data de prescrição inicial e o acompanhamento foi menor para pacientes ERM, } \\
\text { contudo 45,5\% dos pacientes ERM foram trocados para GM, bem como o grupo } \\
\text { ERM levou a maior carga administrativa na maior parte do período analisado. } \\
\text { Estudos prospectivos devem ser conduzidos para confirmar se a conveniência do } \\
\text { paciente, adesão e a acne são realmente melhoradas usando ERM vs GM. }\end{array}$ \\
\hline Patwardhan et al. (2017) & 24 & $\begin{array}{c}\text { Mensurar a gravidade de lesões acneicas usando a } \\
\text { identificação de Coproporfirina III, Protoporfirina } \\
\text { IX, e o grau de inflamação da lesão acneica pelo } \\
\text { aparelho VISIA@-CR, e compará-los com a } \\
\text { avaliação do investigador por meio da contagens } \\
\text { de lesões. Além disso, usar tais métodos para } \\
\text { avaliar os efeitos do tratamento com peróxido de } \\
\text { benzoíla (BPO) 5\%, e a combinação de } \\
\text { Clindamicina + BPO }\end{array}$ & $\begin{array}{l}\text { Tirotricina } 0,1 \% \text { vs BPO } \\
5 \% \text { e combinação de } \\
\text { Clindamicina }+ \text { BPO }\end{array}$ & $\begin{array}{l}\text { A medida da fluorescência de Coproporfirina III e o grau de inflamação das } \\
\text { lesões de acne pelo aparelho VISIA®-CR demonstraram poder de fornecer uma } \\
\text { indicação objetiva da gravidade da acne comedoniana e papulopustulosa, } \\
\text { respectivamente. Além disso, essas medidas demonstraram ser mais sensíveis e } \\
\text { específicas na avaliação dos efeitos do tratamento e primeiros sinais de } \\
\text { progressão da lesão de acne em comparação com a contagem de lesões realizada } \\
\text { pelos investigadores. Em relação aos tratamentos, não houve diferença } \\
\text { significativa observada entre os dois grupos de tratamento. }\end{array}$ \\
\hline Proença (2017) & 5 & $\begin{array}{l}\text { Relatar cinco casos de acne nodular grave, acne } \\
\text { grau IV e acne fulminans, com variante } \\
\text { intensamente inflamatória que precisa ser tratada } \\
\text { com corticosteroides. }\end{array}$ & Prednisona & $\begin{array}{c}\text { Todos os pacientes foram do sexo masculino, entre } 14 \text { e } 21 \text { anos. A melhora dos } \\
\text { sintomas ocorreu durante a primeira semana de tratamento, revertendo as } \\
\text { manifestações clínicas. Os corticosteroides devem ser associados às medidas } \\
\text { locais e sistêmicas de rotina. }\end{array}$ \\
\hline Saviuc et al. (2017) & $\begin{array}{l}\text { Pesquisa in } \\
\text { vitro }\end{array}$ & $\begin{array}{l}\text { Avaliar a atividade antimicrobiana e possíveis } \\
\text { mecanismos de ação de uma combinação de } \\
\text { compostos vegetais a fim de propor uma nova } \\
\text { formulação tópica para tratamento de acne. }\end{array}$ & $\begin{array}{c}\text { Antimicrobianos } \\
\text { derivados de plantas (isto } \\
\text { é, eugenol, -pineno, } \\
\text { eucaliptol e limoneno) e } \\
\text { agentes anti-inflamatórios }\end{array}$ & $\begin{array}{c}\text { Os resultados obtidos revelaram o eugenol / -pineno / 2-fenoxietanol / potássio } \\
\text { sorbato como a combinação antimicrobiana mais potente, atuando em pelo } \\
\text { menos dois agentes microbianos diferentes, isto é, permeabilidade da parede } \\
\text { celular e atividade de bombas de efluxo. Além de sua atividade antimicrobiana, } \\
\text { os compostos naturais incluídos na formulação proposta também são conhecidos } \\
\text { por exibir outras atividades antiacne, como eliminação de radicais livres, } \\
\text { antilipase, anti-inflamatório, e ceratolítico }\end{array}$ \\
\hline $\begin{array}{l}\text { Gold, Weiss, Rueda, Liu e } \\
\text { Tanghetti (2016) }\end{array}$ & 503 & $\begin{array}{c}\text { Avaliar a eficácia e segurança de gel tópico de } \\
\text { adapaleno } 0,3 \% \text { + peróxido de benzoíla 2,5\% em }\end{array}$ & $\begin{array}{c}\text { Combinação em gel de } \\
\text { Adapaleno } 0,3 \%+\text { Gel de }\end{array}$ & $\begin{array}{l}\text { Os resultados deste ensaio clínico demonstram a eficácia significativamente } \\
\text { maior de adapaleno } 0,3 \% \text { + peróxido de benzoíla } 2,5 \% \text { gel tópico em } \\
\text { comparação com o placebo, bem como uma boa segurança no perfil de }\end{array}$ \\
\hline
\end{tabular}


Research, Society and Development, v. 10, n. 4, e39010515094, 2021

(CC BY 4.0) | ISSN 2525-3409 | DOI: http://dx.doi.org/10.33448/rsd-v10i5.15094

\begin{tabular}{|c|c|c|c|c|}
\hline & & $\begin{array}{l}\text { indivíduos com acne inflamatória de moderada a } \\
\text { grave em comparação a gel placebo }\end{array}$ & $\begin{array}{l}\text { peróxido de benzoíla } \\
2,5 \%\end{array}$ & $\begin{array}{l}\text { tratamento de acne inflamatória não nodulocística moderada a grave, o que } \\
\text { aumenta as opções de tratamento dos pacientes, agora com uma concentração } \\
\text { maior de adapaleno. }\end{array}$ \\
\hline $\begin{array}{l}\text { Shemer, Shiri, Mashiah, } \\
\text { Farhi e Gupta (2016) }\end{array}$ & 150 & $\begin{array}{c}\text { Avaliar a segurança, tolerabilidade e eficácia de } 2 \\
\text { doses de espuma tópica de minociclina (1\% e } \\
4 \% \text { ) versus placebo em indivíduos com Acne } \\
\text { Vulgar de moderada a grave }\end{array}$ & $\begin{array}{l}\text { Espuma tópica de } \\
\text { minociclina }(1 \% \text { e } 4 \%)\end{array}$ & $\begin{array}{c}\text { O tratamento com espuma tópica de minociclina } 4 \% \text { levou a uma maior redução } \\
\text { na contagem de lesões versus grupo controle em } 12 \text { semanas. }\end{array}$ \\
\hline $\begin{array}{l}\text { Pan-In, Wongsomboon, } \\
\text { Kokpol, } \\
\text { Chaichanawongsaroj e } \\
\text { Wanichwecharungruang } \\
\text { (2015) }\end{array}$ & 10 & $\begin{array}{l}\text { Avaliar o uso de gel de nanopartículas altamente } \\
\text { carregadas com a-mangostina para tratamento da } \\
\text { acne }\end{array}$ & $\begin{array}{c}\text { Gel de nanopartículas } \\
\text { altamente carregadas com } \\
\text { a-mangostina }\end{array}$ & $\begin{array}{l}\text { Encontrou-se efeito terapêutico das nanopartículas de mangostina em } 10 \\
\text { pacientes com acne usando um estudo randomizado de } 4 \text { semanas, duplo-cego, } \\
\text { controlado por placebo, dupla face. O satisfeito ensaio clínico preliminar obtido } \\
\text { aqui verifica uma nova forma de tratar e também prevenir a acne através da } \\
\text { deposição de mangostina } 400 \mathrm{~nm} \text { nanopartículas nos folículos capilares. }\end{array}$ \\
\hline Richter et al. (2015) & 24 & $\begin{array}{l}\text { Avaliar a eficácia e a tolerabilidade da tirotricina } \\
\text { tópica } 0,1 \% \text { no tratamento da acne em } \\
\text { comparação ao BPO 5\% e à associação da } \\
\text { Clindamicina + BPO }\end{array}$ & $\begin{array}{l}\text { Tirotricina tópica } 0,1 \% \text {, } \\
\text { BPO } 5 \% \text { tópico, } \\
\text { clindamicina }+ \text { BPO }\end{array}$ & $\begin{array}{c}\text { Embora os efeitos do tratamento com tirotricina } 0,1 \% \text { não tenham sido tão fortes } \\
\text { quanto nos comparadores estabelecidos, pode-se concluir que pode ser uma } \\
\text { abordagem alternativa no tratamento da acne leve a acne papulopustulosa grave. } \\
\text { Não é conhecido por causar resistência e parece ser mais tolerável em } \\
\text { comparação às demais terapias (BPO } 5 \% \text { tópico, clindamicina + BPO). Mais } \\
\text { pesquisas são necessárias para estudar o mecanismo de ação, o potencial anti- } \\
\text { inflamatório de tirotricina, efeitos de longo prazo e a eficácia de formulações } \\
\text { alternativas (por exemplo, produtos de combinação). }\end{array}$ \\
\hline
\end{tabular}

Fonte: Barbosa et al. (2021). 
A partir dos trabalhos cuidadosamente selecionados e elencados no Quadro 1, é discutida na próxima sessão, quais os tratamentos empregados no tratamento da acne vulgar em adolescentes e jovens adultos e suas principais implicações.

\section{Discussão}

A acne vulgar é uma doença de etiologia multifatorial e, por isso, diversas terapêuticas precisam ser adotadas para tratar essa doença de forma eficaz. Alguns dos tratamentos medicamentosos disponíveis para acne envolvem: compostos com retinóides, antibióticos, associações de antibióticos e peróxido de benzoíla, associações de antibióticos e retinóides, anticoncepcionais orais e formulações a partir de extratos naturais (Dikicier, 2019; Saviuc et al., 2017; Fuchs et al., 2019).

Retinóides, dentre os quais encontram-se a isotretinoína e a tretinoína, são metabólitos da vitamina A e não possuem totalmente esclarecido seu mecanismo de atuação no tratamento da acne vulgar, contudo, constituem um marco no tratamento dessa condição dermatológica (Soyuduru et al., 2019). A isotretinoína, usada mais comumente para acne vulgar a partir do grau III (papulopustulosa) é de ação sistêmica (Soyuduru et al., 2019), ao passo que a classe possui representantes de uso tópico, como a loção de tretinoína 0,05\%, utilizadas desde o grau de acne comedoniana, ou grau I (Einchefield et al., 2019; Dikicier, 2019).

Eichenfield et al. (2019), analisaram a administração de loção de tretinoína 0,05\% em 74 adolescentes até 13 anos, em sua maioria com acne moderada (cerca de $95 \%$ ), observando melhora significativa, quando comparado ao grupo controle, da semana 8 até a semana 12 de estudo.

Chama-se atenção ainda, para efeitos colaterais do tratamento com a isotretinoína oral. Soyuduru et al. (2019) observaram aumentos estatisticamente significativos nos níveis de colesterol total, LDL-colesterol e triglicerídeos após 5 meses de tratamento com o fármaco, bem como diminuição significativa no HDL-colesterol.

Antibióticos são outra classe largamente empregada no tratamento da acne, sejam em formulações tópicas ou em comprimidos orais. Fundamentam-se no combate ao P. acnes, um dos pilares da fisiopatologia da acne. Dessa forma, objetivando investigar a minociclina, antibiótico usado no tratamento da acne, Gupta et al. (2017) realizaram um estudo comparando a minociclina em uma formulação de liberação prolongada (ERM), uma vez ao dia e uma formulação genérica duas vezes ao dia (GM), observando que, embora ERM seja mais conveniente ao paciente, não ofereceu uma vantagem clínica significativa sobre o GM, apesar do estudo ter apresentado várias limitações, como discrepância nos tamanhos das amostras entre pacientes prescritos com ERM e GM e a alta taxa de abandono ao tratamento com ERM.

Para analisar a eficácia e segurança da espuma de minociclina tópica (FMX-101) em acne vulgar moderada a grave foi realizado um estudo com 139 indivíduos usando FMX-101 4\%, FMX-101 1\% e grupo controle. Observou-se que o tratamento com FMX-101 4\% levou a uma maior redução percentual média das lesões em 12 semanas para lesões inflamatórias e não inflamatórias, se comparado ao grupo controle. Essa redução já pôde ser observada na semana 3 e permaneceu até o final do estudo. Já com FMX-101 1\%, a redução ocorreu apenas para lesões inflamatórias. Nesse cenário, o tratamento com FMX-101 foi bem tolerado e seguro, sem efeitos colaterais sistêmicos ou eventos adversos graves (Shemer et al., 2016).

Apesar de a importância terapêutica dos antibióticos no tratamento de acne vulgar, o aparecimento de cepas bacterianas resistentes, principalmente, à eritromicina e à clindamicina tem causado preocupação. Com o intuito de reduzir o surgimento de tais bactérias, uma opção terapêutica que é capaz de estimular a cicatrização de feridas de pele sem induzir resistência bacteriana é a Tirotricina 0,1\%, um peptídeo antimicrobiano. Assim, um estudo realizado por Richter et al. (2016), comparou a resposta terapêutica entre a tirotricina $0,1 \%$, peróxido de benzoíla (BPO) e uma combinação de clindamicina + BPO. Os resultados do estudos mostraram que a regressão de lesões inflamatórias e não inflamatórias foram mais acentuadas com o uso clindamicina + BPO e BPO, respectivamente. No entanto, a tirotricina também apresentou significativa redução das lesões, além de apresentar o menor índice de efeitos adversos, sendo levantada como mais segura nos tratamentos mais prolongados de acne. 
Em tal panorama, o estudo do potencial de alguns compostos naturais derivados de plantas, quando utilizados em conjunto, representam uma alternativa interessante a ser discutida no tratamento dessa afecção (Saviuc et al., 2017). A partir disso, Saviuc et al. (2017), decidiram testar a eficácia da sinergia de alguns extratos naturais, a saber, eugenol, $\beta$-pineno, 2 fenoxietanol e o sorbato de potássio no combate à acne. Essa composição apresenta propriedades anti-inflamatórias, efeitos relevantes no combate à radicais livres, e, principalmente, na inativação microbiana dos principais patógenos envolvidos no quadro clínico da acne. As potentes propriedades antimicrobianas dessa combinação, descobertas neste estudo, possibilitaram a criação de um novo medicamento, hoje amplamente utilizado no tratamento da acne (Saviuc et al., 2017).

Seguindo a mesma perspectiva, Pan-In et al. (2015) experimentaram o uso de nanopartículas com extrato de mangostão, uma planta comum do sudeste asiático, no tratamento da acne. Essa planta apresenta propriedades antimicrobianas contra o $P$. acnes, ação contra fungos, efeitos importantes na cicatrização de feridas e, também, é anti-inflamatória. O principal componente ativo dessa planta, a alfa-mangostina, quando carreada através de nanopartículas de celulose, pode ser aplicada diretamente no folículo piloso, principal local de ação do P. acnes, sendo relevante como recurso terapêutico dessa afecção (Pan-In et al., 2015). Neste estudo, foi testado em 10 pacientes com acne vulgar o gel de nanopartículas de mangostão. Inicialmente, foi realizada a aplicação de peróxido de benzoíla para preparação da pele, seguida da retirada desse produto, para posterior aplicação do gel, duas vezes ao dia, em apenas um lado da face. Após quatro semanas, os efeitos dessa terapêutica foram positivos, pois houve uma redução de 50\% do número inicial das lesões acneicas após o uso do gel. Assim, através deste ensaio clínico, ficou evidente uma nova possibilidade de tratamento e prevenção da acne (Pan-In et al., 2015).

Segundo Gold et al. (2016), uma outra forma de contribuir para a diminuição do aumento da resistência bacteriana causada pelo uso dos antibiótico é a combinação de retinóide tópico, como o adapaleno 0,1\%, e um antimicrobiano, BPO, que apresentam efeitos sinérgicos superiores ao seu uso individual, além de resumir o tratamento acerca da quantidade de medicamentos a serem ofertados. Ademais, o estudo mostrou que o aumento da concentração de adapaleno para $0,3 \%$ abrangeu o sucesso no tratamento para a acne inflamatória não nodulocística moderada a grave. Dessa forma, apesar do tratamento tópico ainda não ser indicado nesses casos, o sinergismo do adapaleno 0,3\% e BPO prometem mudar esse cenário (Gold et al., 2016).

Outras alternativas para o tratamento dessa doença estão sendo pesquisadas. Karoglan et al. (2019), por exemplo, demonstraram os benefícios do uso de cepas de $P$. acnes em pacientes com acne leve a moderada. Nesse estudo, os 14 pacientes foram submetidos a duas etapas do tratamento. Primeiramente, foi utilizado o peróxido de benzoíla por 7 dias para garantir a redução da microbiota da pele, em seguida, as cepas de P. acnes foram aplicadas topicamente. Essa seleção de cepas benéficas proporcionou melhora das lesões inflamatórias de acne e redução do número de comedões. No entanto, a pequena amostra e a ausência de grupo controle impedem a avaliação da real eficácia e da segurança dessa terapêutica no tratamento da acne (Karoglan et al., 2019).

Patwardhan et al. (2017) realizaram um estudo para avaliar as correlações existentes entre a detecção por fluorescência de substâncias produzidas pelo P. acnes - a coproporfirina III (CpIII) e a protoporfirina IX (PpIX) - e a identificação de lesões acneicas e sua gravidade. Além disso, investigaram acerca do efeito do tratamento com BPO 5\%, comparando-o à associação de clindamicina e BPO e como essa melhora foi demonstrada pelas técnicas de detecção usando CpIII e PpIX. Dessa forma, embora aos 12 dias de tratamento o grupo tratado com BPO 5\% tenha apresentado melhora das lesões comparado ao outro grupo, após 25 dias de tratamento, a contagem de lesões não indicou diferença significativa entre os grupos analisados. A diminuição das áreas de lesões nas pacientes foi seguida pela diminuição da detecção das substâncias, sendo encontrada uma correlação forte com a CpIII e uma correlação fraca com a PpIX.

Ademais, levando em consideração que o hiperandrogenismo é uma das causas de acne vulgar e a contracepção oral auxilia no controle hormonal, as pílulas hormonais antiandrogênicas como a dienogeste, ciproterona, clormadinona, e 
drospirenona, apresentam-se como opções terapêuticas eficazes na redução significativa da intensidade da acne, após três meses de uso em todos os cinco grupos de medicamentos citados (Fuchs et al., 2019).

A acne fulminans, outra variante da acne vulgar, pode apresentar como manifestações iniciais as mesmas lesões elementares da acne grau I e II sendo, no entanto, diferenciada pela presença de cicatrizes deformantes e acometimento sistêmico devido à exacerbada resposta da imunidade adaptativa à bactéria $P$. acne. Para a reversão do quadro de piora súbita da acne, febre alta e artralgia, Proença (2017) afirma que, dentre os casos estudados, todos os pacientes evoluíram com melhora rápida dos sintomas inflamatórios após a administração de doses iniciais de prednisona que variaram de $40 \mathrm{mg} / \mathrm{dia}$ a $60 \mathrm{mg} / \mathrm{dia}$. Além do corticoide, medidas locais e sistêmicas de rotina devem ser associadas.

Um aspecto importante que interfere no manejo adequado de pacientes com acne é a descontinuidade do tratamento. Em estudo com 250 pacientes com acne submetidos a diferentes tratamentos tópicos, Dikicier (2019) observou que 114 (45,6\%) dos participantes abandonaram o tratamento antes do período estipulado. Como razões para o abandono da terapêutica, os participantes citaram falta de resposta (62,3\%) e efeitos colaterais $(37,7 \%)$ como irritação, eritema, descamação, prurido e ardência. A taxa de descontinuidade do tratamento foi significativamente maior naqueles pacientes com acne grave (Dikicier, 2019).

Nessa perspectiva, a adoção de uma terapêutica coadjuvante que diminua esses efeitos colaterais pode ser uma alternativa eficaz para melhoras a adesão dos adolescentes e jovens adultos ao tratamento. Pensando nisso, Monfrecola et al. (2018) experimentou o uso do creme facial acne RA-1,2, o qual contém filtro solar, Salix alba, 1,2-decanediol, vitaminas B3, C e E e isoflavonoides de soja, associado ao tratamento farmacológico padrão da acne uma vez ao dia, por três meses em quarenta pacientes. Esse medicamento é seletivo contra a radiação UV e as suas propriedades fotoprotetoras auxiliam na redução da produção de sebo e da perda hídrica, além de reduzir os efeitos adversos do tratamento farmacológico, por aumentar a tolerabilidade da pele. Essa combinação proporcionou uma adesão completa dos pacientes à terapêutica medicamentosa instituída, evidenciando, após 3 meses, redução significativa do quadro clínico de acne (comedoniana e papulopustular) considerando os escores da avaliação global do investigador (Monfrecola et al., 2018).

\section{Conclusão}

A acne vulgar, por se tratar de uma doença multifatoral, e por ter formas clínicas com variáveis graus de inflamação, possui muitas formas de tratamento disponíveis. Os tratamentos medicamentosos têm sido aprimorados e diversificados, podendo envolver desde a administração medicamentosa tópica à oral, uso de nanopartículas, contraceptivos, corticosteroides, substâncias de proteção UV, extratos originados da natureza, terapias combinadas. No entanto, ressalva-se a necessidade de mais estudos científicos a fim de desenvolver tratamentos mais eficazes e com menos efeitos colaterais, contribuindo para e maior adesão dos adolescentes e jovens adultos acometidos aos tratamentos.

\section{Referências}

Almeida-Junior, S., Cruz, R. C. R., Castellane, J. A. S., Marrafon, A. A., Gomes, S. F. A., Oliveira, K. R. P., \& Furtado, R. A. (2020). Application of Melaleuca alternifolia essential oils associated with phototherapy in acneic inflammatory injury. Research, Society and Development, 9(11), e9889119856. https://doi.org/10.33448/rsd-v9i11.9856

Bagatin, E., Timpano, D. L., Guadanhim, L. R. S., Nogueira, V. M. A., Terzian, L. R., Steiner, D., \& Florez, M. (2014). Acne vulgaris: prevalence and clinical forms in adolescents from São Paulo, Brazil. Anais Brasileiros de Dermatologia, 89(3), 428-435. https://doi.org/10.1590/abd1806-4841.20142100

Costa, F. V., Souza, L. S., Conradesque, P. R., Carniel, P., \& Zago, A. (2020). Indução percutânea de colágeno como tratamento de cicatrizes da Acne vulgar: uma revisão integrativa. Research, Society and Development, 9(8), e252985706. https://doi.org/10.33448/rsd-v9i8.5706.

Costa, I. V., \& Velho, G. M. C. C. (2018). Acne Vulgar no Adulto. Revista Sociedade Portuguesa de Dermatologia e Venereologia, 76(3), 299-312. https://dx.doi.org/10.29021/spdv.76.3.953 
Dikicier, B. S. (2019). Topical treatment of acne vulgaris: efficiency, side effects, and adherence rate. Journal of International Medical Research, 47(7), 29872992

Eichenfield, L. F., Sugarman, J. L., Guenin, E., Harris, S., \& Bhatt, V. (2019). Novel tretinoin $0.05 \%$ lotion for the once-daily treatment of moderate-to-severe acne vulgaris in a preadolescent population. Pediatric Dermatology, 36,193-199.

Fuchs, A., Matonog, A., Sieradzka, P., Pilarska, J., Hauzer, A., Czech, I., \& Drosdzol-Cop, A. (2019). Anti-androgenic therapy in Young patients and its impact on intensity of hirsutism, acne, menstrual pain intensity and sexuality — a preliminary study. Ginekologia Polska, 90(9), 520-526.

Garcia, A. K. A., Fonseca, L. F., Aroni, P., \& Galvão, C. M. (2016). Estratégias para o alívio da sede: revisão integrativa da literatura. Revista Brasileira de Enfermagem, 69(6), 1148-55.

Gold, L. S., Weiss, J., Rueda, M. J., Liu, H., \& Tanghetti, E. (2016). Moderate and Severe Inflammatory Acne Vulgaris Effectively Treated with Single-Agent Therapy by a New Fixed-Dose Combination Adapalene 0.3 \%/Benzoyl Peroxide 2.5 \% Gel: A Randomized, Double-Blind, Parallel-Group, Controlled Study. Am J Clin Dermatol, 17, 293-303.

Gupta, P., Timothy S., Sopkovich, J., Massick, S., \& Kaffenberger, B. H. (2017). Generic twice daily minocycline vs. branded extended-release minocycline for acne: a retrospective comparison of treatment escalation. J Am Acad Dermatol, 76(6), 1195-1197.

Karoglan, A., Paetzold, B., Lima, J. P., Brüggemann, H., Tüting, T., Schanze, D., Güell, M., \& Gollnick, H. (2019). Safety and efficacy of topically applied selected cutibacterium Acnes strains over five weeks in patients with acne vulgaris: an Open-label, pilot study. Acta derm venereol, 99, $1243-1257$.

Lima, M. F. S, Barros, V. J. S, \& Lúcio, M. P., \& Neto. (2020). Analysis of oral isotretinoin consumption in the specialized component of pharmaceutical assistance in the state of Piauí. Research, Society and Development, 9(2), e170922235. https://doi.org/10.33448/rsd-v9i2.2235

Montagner, S., Costa, A. (2010). Guidelines in the treatment of acne. Surgical \& Cosmetic Dermatology, 2(3), 205-213.

Monfrecola, G., Capasso, C., Russo, G., \& Fabbrocini, G. (2018). UV-selective face cream (Acne RA-1,2) in acne patients: clinical study of its effects on epidermal barrier function, sebum production, tolerability and therapy adherence. Giornale Italiano di Dermatologia e Venereologia, 153(1), $26-32$.

National Center for Biotechnology Information - NCBI (2009). Young adult. MeSH terms. Recuperado em 3 fevereiro, 2021, de https://www.scielo.br/scielo.php?script=sci_arttext\&pid=S0365-05962014000300428.

Pan-In, P., Wongsomboon, A., Kokpol, C., Chaichanawongsaroj, N., \& Wanichwecharungruang, S. (2015). Depositing a-mangostin nanoparticles to sebaceous gland área for acne treatment. Journal of Pharmacological Sciences, 129, 226-232.

Patwardhan, S. V., Richter, C. Vogt, A., Blume-Peytavi, U., Canfield, D., \& Kottner, J. (2017). Measuring acne using Coproporphyrin III, Protoporphyrin IX, and lesion-specific inflammation: an exploratory study. Arch Dermatol Res, 309, 59-167.

Pavithra, G., Upadya, G. M., \& Rukmini, M. S. (2019). A randomized controlled trial of topical benzoyl peroxide $2.5 \%$ gel with a low glycemic load diet versus topicalbenzoyl peroxide 2.5\% gel with a normal diet in acne (grades 1-3). Indian Journal of Dermatology, Venereoly and Leprology, 85, $486-90$.

Proença, N. G. (2017). Acne fulminans. Anais Brasileiros de Dermatologia, 92, 8-10.

Richter, C., Trojahn, C., Hillmann, K., Dobos, G., Stroux, A., Kottner, J., \& Blume-Peytavi, U. (2016). Reduction of Inflammatory and Noninflammatory Lesions with Topical Tyrothricin $0.1 \%$ in the Treatment of Mild to Severe Acne Papulopustulosa: A Randomized Controlled Clinical Trial. Skin Pharmacol Physiol, 29, 1-8.

Rodrigues, F. M., Leite, R. S., Yoshida, E. H., Carneiro, F. P., \& Santos, N. S. (2019). Tratamento dermatológico da acne vulgar. Revista Saúde em Foco, 11 , 339-345.

Santos, L. S., Martins, P. C. M. L., \& Perez, F. S. B. Terapia de indução de colágeno no tratamento de cicatrizes de acne: revisão de literatura. Saúde \& Ciência em Ação, 5(01), 98-110.

Saraiva, T. A., Souza, L. S., Costa, K. F., Leroy, P. L. A., \& Hermínio, M. R. S. (2020). A laserterapia no tratamento da acne vulgar. Revista Brasileira Militar de Ciências, 6(15), 59-66.

Saviuc, C., Ciubucã, B., Dincã, G., Bleotu, C., Drumea, V., Chifiriuc, M., Popa, M., Pircalabioru, G. G., Marutescu, L., \& Lazãr, V. (2017). Development and Sequential Analysis of a New Multi-Agent, Anti-Acne Formulation Based on Plant-

Derived Antimicrobial and Anti-Inflammatory Compounds. International Journal of Molecular Sciences, 18, 175.

Shemer, A., Shiri, J., Mashiah, J., Farhi, R., \& Gupta, A. K. (2016). Topical minocycline foam for moderate to severe acne vulgaris: Phase 2 randomized doubleblind, vehicle-controlled study results. Journal of de American Academy of Dermatology, 74(6), 1251-2.

Silva, J. C., \& Pereira, P. C. (2018). Avaliação e tratamento estético da acne vulgar. Revista Científica Universitas, 5(1), 114-123.

Silva, M. C. J., Nascente, F. M., Souza, C. M. D., Cardoso, A. M., Ferreira, L. D. L. P., \& Sobrinho, H. M. R. (2020). Os benefícios da limpeza de pele no tratamento coadjuvante da acne vulgar. Revista Brasileira Militar de Ciências, 6(16), 28-36.

Souza, M. T., Silva, M. D., \& Carvalho, R. (2010). Revisão integrativa: o que é e como fazer. Einstein, 8(1), 102-106.

Soyuduru, G., Adişen, E. Ö., Özer, İ., \& Aksakal, A. B. (2019). The effect of isotretinoin on insulin resistance and adipocytokine levels in acne vulgaris patients. Turkish journal of medical sciences, 49(1), 238-244. 